\title{
LEVANTAMENTO EPIDEMIOLÓGICO DA DENGUE NO ESTADO DO PARANÁ BRASIL NOS ANOS DE 2011 A 2015
}

\author{
Gisele da Silva Brigagão ${ }^{1}$ \\ Nelton Anderson Bespalez Corrêa ${ }^{2}$
}

BRIGAGÃO, G. da S.; CORRÊA, N. A. B. Levantamento epidemiológico da dengue no estado do Paraná Brasil nos anos de 2011 a 2015. Arq. Cienc. Saúde UNIPAR, Umuarama, v. 21, n. 1, p, 41-45, jan./abr. 2017.

\begin{abstract}
RESUMO: Este estudo tem como objetivo descrever os dados epidemiológicos da dengue no Estado do Paraná, no período de 2011 a 2015 , por meio da análise dos números de casos. Considerada a principal doença reemergente nos países tropicais e subtropicais, transmitida pelo mosquito Aedes aegypti, a dengue vem sendo um grave problema de saúde pública no Brasil. O Estado do Paraná tem apresentado aumento significativo nos números de casos notificados nos últimos anos analisados, em que o período de 2012/2013 contabilizou os maiores índices com um aumento considerável de 366,18\% quando comparado ao período 2011/2012 de menor índice e de $1.943 \%$ quanto ao número de casos confirmados, com incidência de 498,62, situação de epidemia ( $\geq 300$ casos/100.000 hab.). O período de 2015/2016 apresentou um número alarmante de 61 óbitos por dengue, sendo um aumento de $6.000 \%$ comparado ao período de menor número em 2011/2012, um dado preocupante, indicativo do quanto vem aumentando os casos de dengue grave com evolução para óbito. A idade mais acometida tem compreendido a faixa etária entre 20-49 anos, diante disto e dos números crescentes, o governo investiu em 2016 na vacina contra a dengue, disponibilizando a primeira dose da vacina, em 30 municípios paranaenses, nos quais se concentraram $80 \%$ dos casos, tendo inicialmente como público alvo a faixa etária de 15 a 27 anos, na tentativa de reduzir os números registrados da dengue.
\end{abstract}

PALAVRAS-CHAVE: Dengue. Epidemiologia. Dengue/Paraná.

\section{DENGUE EPIDEMIOLOGICAL SURVEY IN THE STATE OF PARANÁ, BRAZIL FROM 2011 TO 2015}

\begin{abstract}
This study aims to describe the epidemiological data on dengue fever in Paraná, from 2011 to 2015, by analyzing the number of reported cases. Considered the main reemerging disease transmitted by the Aedes aegypti mosquito in tropical and subtropical countries, dengue fever has been a major public health issue in Brazil. Paraná has shown a significant increase in the numbers of cases reported in recent years, with the $2012 / 2013$ period recording the highest rates, with a considerable increase of $366.18 \%$ compared with the period of lowest rates, 2011/2012 and 1,943\% on the number of confirmed cases, with an incidence of 498.62 cases, which is considered an epidemic situation ( $\geq 300$ cases/100,000 inhabitants). The 2015/2016 period showed an alarming number of 61 deaths from dengue fever, with a $6,000 \%$ increase compared to the period of 2011/2012 which presented the lowest rates. This is a concern, since it is an indicative of increased cases of severe dengue progressing to death. The most affected age group was that of 20-49 years old, due to this and to the increasing numbers, the state government invested in dengue fever vaccine in 2016, providing the first dose to 30 municipal districts that concentrated $80 \%$ of cases, initially targeting the age group of 15-27 years, in an attempt to reduce the registered numbers of dengue fever. KEYWORDS: Dengue fever. Dengue fever/Paraná. Epidemiology.
\end{abstract}

\section{Introdução}

Uma importante causa de morbidade no Brasil e no Mundo é a presença de doenças transmitidas por vetores, como os insetos. A incidência de algumas doenças transmitidas por insetos gera preocupação devido ao aquecimento global em países de clima temperado, onde fatores de ordem biológica, geográfica, ecológica, social, cultural e econômica atuam sinergicamente na produção, distribuição e controle destas doenças (TAUIL, 2002).

Considerada a principal doença reemergente nos países tropicais e subtropicais, a dengue é um vírus transmitido principalmente pelo mosquito Aedes aegypti, sendo um grave problema de saúde pública no Brasil, assim como em outras regiões tropicais do mundo, devido apresentar enorme facilidade de adaptação a diversas condições ambientais (MARZOCHI, 1994; TAUIL, 2006).

O mosquito Aedes aegypti deposita seus ovos em locais com água parada como em tanques, baldes, potes, latas, vasos de flores, pias, calhas e caixas de água, ou em qualquer lugar onde se possa acumular água limpa (TAUIL, 1987). O ciclo reprodutivo do Aedes aegypti é influenciado pela variação de temperatura, em que a incidência sazonal se dá nos meses quentes, verificando que a incidência de novos casos de dengue diminui significativamente nos meses mais frios, na segunda metade do ano, contudo, a transmissão da doença não é interrompida neste período (CONSOLI; OLIVEIRA, 1994).

Apesar de se observar casos registrados nas diversas classes sociais, manifestados em parcelas da população de alta, média e de baixa renda, a dengue atinge em maior proporção populações de maior carência socioeconômica e habitantes de periferias urbanas já que está dentro desta camada social os principais índices de letalidade decorrentes de complicações relacionadas à dengue (MENDONÇA; PAULA; OLIVEIRA, 2004). O saneamento básico, particularmente, o abastecimento de água e a coleta de lixo, são insuficientes ou inadequados nas periferias das cidades, sendo um dos fatores responsáveis ao favorecimento da proliferação do principal mosquito vetor da dengue, afetando assim esta parte da população de maior carência socioeconômica (GUBLER; KUNO, 1997).

Os primeiros registros de dengue no Estado do Paraná se deram no ano de 1993, a partir de 1995 foram registradas importantes epidemias da doença no Estado (MENDONÇA; PAULA; OLIVEIRA, 2004). Estão presentes no Estado os 4 sorotipos virais da dengue (DEN-1, DEN-2, DEN-3 E DEN-4), sendo o de maior prevalência o sorotipo

DOI: https://doi.org/10.25110/arqsaude.v21i1.2017.6075

${ }^{1}$ Farmacêutica (UNIPAR); giselebrigagao@gmail.com

${ }^{2}$ Docente Curso de Farmácia da Universidade Paranaense (UNIPAR); Praça Mascarenhas de Moraes, 4.282, CEP: 87502-210 Umuarama - Pr, Brasil; nelton@unipar.br 
DEN-1 (PARANÁ, 2013).

Segundo o Ministério da Saúde (2016), o Brasil adotou em 2014 uma nova classificação de dengue, como sendo uma doença única, sistêmica e dinâmica de amplo espectro clínico na dengue sintomática, significando que a doença pode evoluir para uma melhora ou agravamento, podendo evoluir para óbito, o que sugere atenção a quaisquer sintomas para que sejam tomados os devidos cuidados de imediato, na tentativa de reduzir os casos de agravamento e óbitos. Três fases clínicas podem ocorrer: febril, crítica e de recuperação.

A doença ganhou destaque nas estratégias de prevenção mediante a realização de campanhas, dentre as doenças de alta incidência em território brasileiro (PENNA, 2003). Como medidas de prevenção, o Governo do Estado do Paraná impôs seu foco no combate ao mosquito Aedes aegypti, também transmissor dos vírus da Zika e Chikungunya e criou o programa estadual VigiaSUS, para auxiliar as prefeituras na estruturação do setor de vigilância em saúde, com repasse de verbas, na capacitação profissional dos agentes, ações de vigilância ambiental e epidemiológica, vistorias a domicílios com orientação e remoção de possíveis criadouros e campanhas por meio de impressos, rádio e televisão (PARANÁ, 2015).

Faz se necessário o acompanhamento dos números de casos notificados, confirmados, e com agravos da dengue, a fim de avaliar o impacto da doença sob a população, o que pode reorientar as estratégias de combate à doença. Nesta perspectiva o presente estudo apresenta um levantamento epidemiológico da dengue no Estado do Paraná, no período de 2011 a 2015.

\section{Material e Métodos}

Tratou-se de um estudo retrospectivo, descritivo, utilizando como fonte de informações, o banco de dados da Sala de Situação da Dengue/SVS/SESA, por meio de informes técnicos, disponibilizados pelo portal online Dengue Paraná da Secretária de Estado de Saúde do Paraná, que desde o ano de 2011 leva em conta a curva epidemiológica da doença, que geralmente apresenta seu pico durante o verão, sendo assim, os dados começam a ser analisados na semana 31 do mês de agosto e finalizados na semana 30 do mês de julho do ano seguinte.

Foram analisados dados relativos ao Estado do Paraná, selecionados os períodos epidemiológicos de dengue entre os anos de 2011 a 2015, discutindo-se os números de casos notificados suspeitos de dengue, confirmados, com sintomas de risco (casos com sinais de alarme e casos de dengue grave), óbitos e idade acometida.

\section{Resultados e Discussão}

Quanto aos casos de dengue no Estado do Paraná, dos períodos analisados, 2011/2012 apresentou os menores números de casos notificados e confirmados, com incidência considerada baixa (menor que 100 casos/100.000hab.), segundo o Ministério da Saúde (1977). O período de 2012/2013 contabilizou os maiores índices, com um aumento considerável de $366,18 \%$ em número de casos notificados e de $1.943 \%$ em casos confirmados de dengue, quando comparado ao período 2011/2012 de menores índices, colocando o estado em situação de epidemia ( $\geq 300$ casos/100.000 hab.). O período de 2015/2016 apresentou um salto alarmante de $6.000 \%$ nos casos que evoluíram para óbito, quando comparado ao período de 2011/2012 que apresentou o menor número do período (Tabela 1), um dado preocupante, indicativo do quanto vem aumentando os casos de dengue grave com evolução para óbito.

Estudo realizado compreendendo a região Sul do país, demonstra que os maiores índices de casos notificados, de morbidade hospitalar por dengue clássica e de formas graves de dengue se deram no Estado do Paraná, nos anos de 2001 a 2005 (KOYAMA; BALDISSERA, 2009), o que demanda identificação precoce dos casos para implantação de medidas oportunas em relação ao controle da doença, já que quanto antes o diagnóstico melhores as chances de reduzir os índices dos agravamentos e de óbitos por dengue, de acordo com o novo conceito da doença exposto no Plano de Enfrentamento das Doenças provocadas pelo Aedes aegypti (BRASIL, 2016).

Tabela 1: Número de casos de dengue notificados, dengue com sinais de alarme (DSA), dengue grave (DG), óbitos e incidência por 100.000 habitantes, Paraná - 2011 a 2015

\begin{tabular}{|c|c|c|c|c|c|c|c|c|c|}
\hline \multirow{2}{*}{ Período } & \multirow{2}{*}{ População } & \multicolumn{3}{|c|}{ Casos } & \multirow{2}{*}{ Notificados } & \multirow{2}{*}{ Dsa } & \multirow{2}{*}{ Dg } & \multirow{2}{*}{ Óbitos } & \multirow{2}{*}{ Incidência } \\
\hline & & Autóctones & Importados & Total & & & & & \\
\hline $2011 / 2012$ & 10.439 .601 & 2.400 & 278 & 2.678 & 23.762 & 4 & 16 & 1 & 22,99 \\
\hline $2012 / 2013$ & 10.439 .601 & 52.054 & 2.662 & 54.716 & 110.774 & 82 & 147 & 23 & 498,62 \\
\hline $2013 / 2014$ & 10.997 .462 & 18.741 & 887 & 19.628 & 57.075 & 200 & 44 & 9 & 170,41 \\
\hline $2014 / 2015$ & 10.997 .462 & 33.702 & 1.731 & 35.433 & 89.968 & 354 & 122 & 24 & 306,45 \\
\hline $2015 / 2016$ & 11.163 .018 & 52.708 & 3.643 & 56.351 & 145.757 & 780 & 132 & 61 & 472,17 \\
\hline
\end{tabular}

Fonte: Sala de Situação da Dengue/SVS/SESA

No Brasil, a transmissão da dengue ocorre de forma continuada desde 1986, intercalando com a ocorrência de epidemias associadas à introdução de novos sorotipos ou alteração do sorotipo predominante. O maior surto no Brasil ocorreu em 2015, com aproximadamente 1,6 milhões de casos prováveis da doença (BRASIL, 2016). No Estado do
Paraná, em relação ao mesmo período, a incidência se encontrou em situação de epidemia ( $\geq 300$ casos/100.000 hab.), chegando perto do maior índice dentre os períodos analisados, observado em 2012/2013.

Segundo o Ministério da Saúde (2016), deve-se notificar todo caso suspeito de dengue, onde considera-se como 
suspeito de infecção, indivíduos que apresentem relato de febre, usualmente entre dois e sete dias de duração e duas ou mais das seguintes manifestações: náusea, vômitos; exantema; mialgias, artralgia; cefaleia, dor retro-orbital; petéquias; e laboratoriais: prova do laço positiva e leucopenia. Também pode ser considerado caso suspeito toda criança com quadro febril agudo, usualmente entre dois e sete dias de duração, e sem foco de infecção aparente.

Verifica-se por meio da Figura 1, que nos últimos três períodos analisados houve um crescimento consecutivo quanto a notificação dos casos suspeitos de dengue, apresentando um crescimento total dentro do período analisado de $513,49 \%$ de casos notificados.

Estudo desenvolvido em Guaraí no Tocantins, região norte do país, mostrou redução consecutiva anual nos números de casos notificados por dengue no período que compreende 2011 a 2015 , em que reduziu $60 \%$ dos casos notificados no período de 2013 a 2014 (AGUIAR et al., 2016), divergindo da situação do Estado do Paraná, onde houve um aumento de 57,68\% para o período de 2013 a 2014. Em relação a incidência por 100.00 habitantes, o boletim epidemiológico nacional de dengue da Secretaria de Vigilância em Saúde, apresentou redução de 55,39\% para o Estado do Tocantins visto o mesmo período, enquanto o Paraná apresentou uma redução maior, sendo de $63,71 \%$ (BRASIL, 2014).

Figura 1: Percentual de notificações dos casos suspeitos de dengue, Paraná - 2011 a 2015

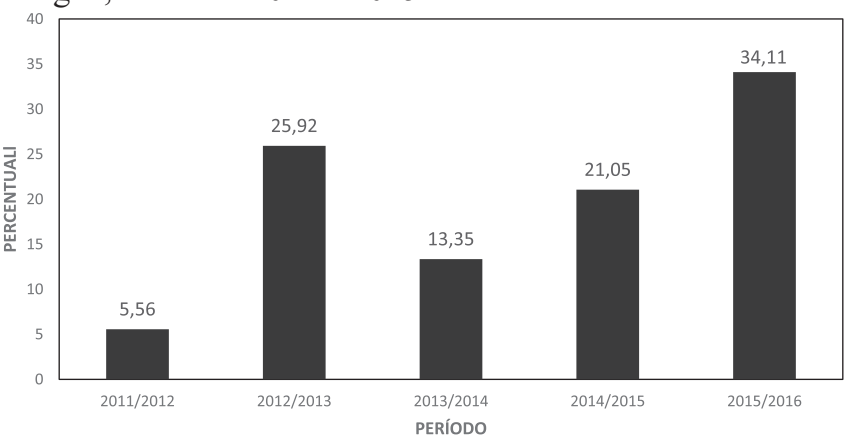

Fonte: Sala de Situação da Dengue/SVS/SESA

É importante considerar, que em março do ano de 2015, foi detectado a entrada de um novo vírus no país na região nordeste, em meio ao surto de dengue na qual o país estava enfrentando, o Zika vírus, em que pacientes infectados foram tratados inicialmente como sendo dengue, até a detecção laboratorial do Zika (CAMPOS; BANDEIRA; SARDI, 2015). O aumento do número de casos notificados pode estar diretamente relacionado com a entrada do vírus da Zika no Brasil em 2015 e também com a entrada do vírus Chikungunya no ano de 2014, também transmitidos pelo vetor do vírus da dengue, acarretando na intensificação das campanhas de combate ao mosquito Aedes aegypti direcionadas a conscientização da população, fato que levou à maior procura das unidades de saúde em busca de diagnóstico correto e precoce, já que estas doenças apresentam sintomas semelhantes como se pode observar por meio da Figura 2.
Figura 2: Folder com a comparação dos sintomas do vírus da Dengue, Zika vírus e Chikungunya, disponibilizado para campanhas pelo Conselho Regional de Farmácia do Estado do Paraná, Paraná - 2016

DIFERENÇA ENTRE AS DOENÇAS DENGUE, ZIKA ECHIKUNGUNYA

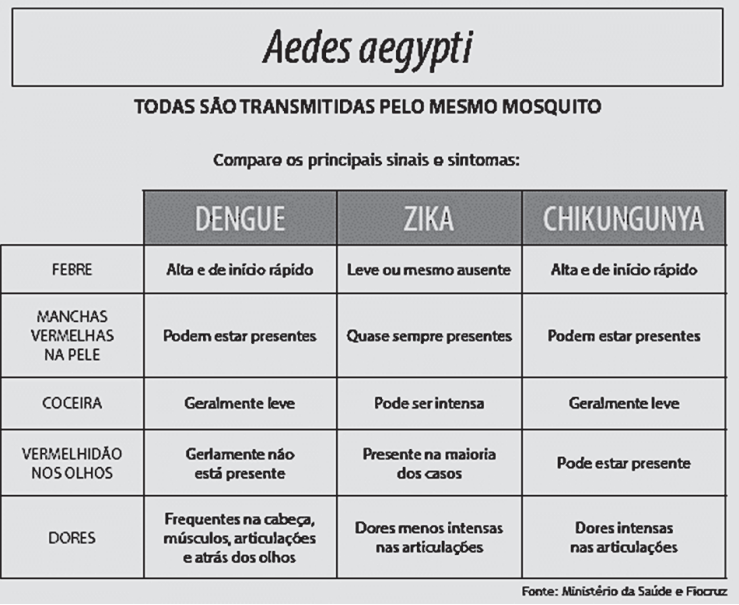

Fonte: Conselho Regional de Farmácia do Paraná

Outro fator que vale observar é a relação da idade acometida no período estudado (Figura 3), na qual destacou-se que mais da metade dos números confirmados de dengue se concentraram nas idades de 20 a 49 anos, faixa etária esta que se trata de uma grande parte da população considerada economicamente ativa (IBGE, 2015).

Estudo de Monteiro (2016), que analisou a dengue no estado do Rio Grande do Norte no período de 2014/2015, apresentou as faixas etárias mais atingidas entre 11-20 e 5160 anos, divergindo da faixa etária mais atingida no Estado do Paraná, mostrando um comportamento distinto entre as extremidades do país.

Figura 3: Distribuição de casos confirmados de dengue por faixa etária, em uma média dos períodos analisados, compreendendo a semana epidemiológica de 31/2011 a 30/2016, Paraná - 2011 a 2016

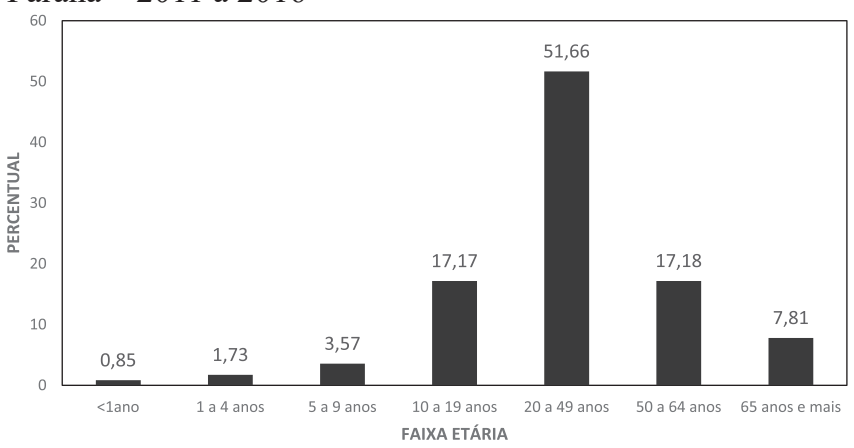

Fonte: Sala de Situação da Dengue/SVS/SESA

Frente ao crescimento da incidência de dengue desde 2013, assim como dos números de dengue com sintomas de risco, o Estado do Paraná se viu na posição de buscar novas estratégias de combate à doença, tornando se o pioneiro a obter a vacina contra a dengue no sistema público de saúde, visando a imunizar em torno de 500 mil pessoas, por meio da administração em três doses com intervalos de seis meses en- 
tre as aplicações, contra os quatro sorotipos virais da doença (PARANÁ, 2016a). A campanha de vacinação iniciou-se em agosto de 2016, disponibilizando a primeira dose da vacina, em 30 municípios paranaenses, dos quais se concentraram $80 \%$ dos casos de dengue registrados no Estado, tendo inicialmente como público alvo a faixa etária de 15 a 27 anos (PARANÁ, 2016).

Diante dos meios já existentes de combate à dengue somados a nova estratégia adotada, se espera observar uma redução nos números da doença no fechamento do próximo período no ano de 2017, nas cidades que receberam as doses da vacina, onde poderão ser realizados novos estudos, possibilitando analisar a eficácia desta ação, incentivando o Estado a ampliar as doses ofertadas da vacina contra a dengue.

É importante salientar que apesar dos esforços, as campanhas de controle da dengue são dificultadas devido sua ocorrência nos grandes centros ser facilitada pelas condições urbanas encontradas, diante da grande variedade de criadouros artificiais disponíveis ao vetor (TAUIL, 2001). Devido a isto, ressalva o secretário da Saúde do Paraná, Michele Caputo Neto que, "Todos têm tarefas na eliminação dos criadouros do mosquito transmissor. O poder público deve manter a vigilância, e todo cidadão deve vistoriar sua casa, trabalho ou vizinhança a procura de água parada. O perigo é real e triplicou" (PARANÁ, 2015).

\section{Conclusão}

Fica evidente, diante do levantamento dos dados, que após a grande epidemia sofrida no Paraná no período de 2012/2013, a dengue veio ganhando forças apresentando aumento anual consecutivo dos casos notificados e os números de casos agravados de dengue, principalmente os que acarretaram em óbito que dão o alerta sob o amplo espectro clínico que a doença tem apresentado.

Considerando os dados analisados, fica claro que as estratégias que vêm sendo utilizadas não estão trazendo bons resultados no controle da disseminação da dengue no Paraná. Com isto, abre-se espaço para que novos estudos reavaliem e reorientem estas estratégias, a fim de controlar esta doença que hoje é um grave problema de saúde pública no Brasil.

Por todas essas razões, faz se necessário a população se conscientizar e somar forças aos programas do governo, permitindo a entrada dos fiscais nos terrenos para vistorias, descartando corretamente os resíduos, denunciando qualquer irregularidade que possa favorecer a proliferação do mosquito Aedes aegypti para Secretária de Saúde do município e quando dentro da faixa etária abrangente a campanha de vacinação, se dirigindo as unidades de saúde que fornecem as doses da vacina, dessa forma, as chances de se alcançar bons resultados na redução nos números de casos de dengue são intensificadas.

\section{Referências}

AGUIAR, J. D. et al. Análise Do Perfil Epidemiológico Dos Casos De Dengue No Município De Guaraí-TO. Revista

Eletrônica de Farmácia, v. 13, n. 1.1, 2016.

BRASIL. Câmara dos Deputados Brasília, Comissão Zika Vírus e Microcefalia. Plano de Enfrentamento das Doenças provocadas pelo Aedes aegypti (Situação epidemiológica e Gestão), 2016. Disponível em: <http://www2.camara.leg. br/atividade-legislativa/comissoes/comissoes-temporarias/ externas/55a-legislatura/acompanhamento-das-acoes-sobreo-zika-virus/documentos/audiencias-publicas/apresentacaoneilton>. Acesso em: 04 jul. 2016.

Ministério da Saúde. Secretaria de Vigilância

em Saúde, Boletim Epidemiológico, v. 45, n. 31, 2014. Disponível em: <http://portalsaude.saude.gov.br/images/ pdf/2015/janeiro/02/2014-039---Dengue-SE-47.pdf $>$. Acesso em: 01 out. 2016.

.Secretaria de Vigilância em

Saúde, Departamento de Vigilância das Doenças Transmissíveis. Dengue: diagnóstico e manejo clínico: adulto e criança. - 5. Ed. - Brasilia, 2016. Disponível em: <http://www.saude.go.gov.br/public/media/

ZgUINSpZiwmbr3/10900120219262619909.pdf > . Acesso em: 01 ago. 2016.

. Secretária Nacional de ações

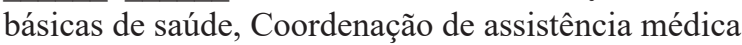
hospitalar. Conceitos e definição em Saúde. Brasilia, 1977. Disponível em: <http://bvsms.saude.gov.br/bvs/ publicacoes/0117conceitos.pdf>. Acesso em: 06 jul. 2016.

CAMPOS, G. S.; BANDEIRA, A. C.; SARDI, S. I. Zika virus outbreak, Bahia, Brazil. Emerging infectious diseases, v. 21, n. 10, p. 1885, 2015.

CONSOLI, R. A. G. B.; OLIVEIRA, R. L. de. Principais mosquitos de importância sanitária no Brasil. Rio de Janeiro: Fio Cruz, 1994.

\section{GUBLER, D. J.; KUNO, G. Dengue and dengue}

hemorrhagic fever. New York: CAB international, cap. 1, p. 1-22, 1997.

IBGE - Instituto Brasileiro de Geografia e estatística. Sistema IBGE de Recuperação Automática - SIDRA. População Economicamente Ativa - PEA, 2015. Disponível em: <http://www.sidra.ibge.gov.br/bda/emprego/default.asp $? \mathrm{t}=2 \& \mathrm{z}=\mathrm{t} \& \mathrm{o}=6 \& \mathrm{u} 1=26674 \& \mathrm{u} 2=26674 \& \mathrm{u} 3=26674 \& \mathrm{u} 4=26$ $674 \& u 5=26674 \& u 6=6567>$. Acesso em: 18 out. 2016.

KOYAMA, A. M.; BALDISSERA, V. D. A. Descrição dos casos de dengue na região sul do Brasil, de 2001 a 2005. Arq. Ciênc. Saúde UNIPAR, Umuarama, v. 13, n. 3, p. 125-132, maio/ago. 2009.

MARZOCHI, K. B. F. Dengue in Brazil: situation, transmission and control - a proposal for ecological control. Mem. Inst. Oswaldo Cruz, v. 89, p. 235-245, 1994.

MENDONÇA, F.; PAULA, E. V.; OLIVEIRA, M. M. F. Aspectos sócio-ambientais da expansão da dengue no Paraná. In: Encontro da Associação Nacional de Pós Graduação e Pesquisa em Ambiente e Sociedade, 2., 2004, São Paulo. Anais... São Paulo: ANPPAS, p. 1-13, 2004. Disponível em: 
$<$ http://www.anppas.org.br/encontro_anual/encontro2/GT/

GT12/anpas_dengue.pdf>.Acesso em: 01 jul. 2016

MONTEIRO, J. D. Epidemiologia molecular dos vírus dengue e zika no estado do Rio Grande do Norte, no período de junho de 2014 a maio de 2015. 2016. $110 \mathrm{f}$. Dissertação (Mestrado em Biologia Parasitária) - Centro de Biociências, Universidade Federal do Rio Grande do Norte, Natal, 2016.

PARANÁ. Secretaria de Estado da Saúde. Situação epidemiológica da dengue. 2013. Disponível em: <http:// slidegur.com/doc/1414495/apresenta $\% C 3 \%$ A7\%C3\%A3odo-powerpoint---conselho-estadual-de-sa\%C3\%BAde>. Acesso em: 07 jun. 2016.

Situação da dengue, Chikungunya e Zika vírus no Paraná - 2015/2016. Curitiba, 2015. Disponível em: <http://www.dengue.pr.gov.br/arquivos/File/ Dengue_Informe_Tecnico_03_2015_2016_SE_49_ZIKA CHIKUNGGUNYĀcomIIP_2015_12_15_final.pdf $>$. Acesso em: 10 ago. 2016.

Situação da dengue, Chikungunya e Zika vírus no Paraná - 2015/2016. Curitiba, 2016. Disponível em: $<$ http://www.dengue.pr.gov.br/arquivos/File/Den gueInformeTcnico35_2015_2016atSE302016_ZIKA_ CHIKUNGUNYA_2016_08_02.pdf $>$. Acesso em: 13 ago. 2016.

Vacina da dengue: perguntas e respostas. 2016. Disponível em: < http://www.saude.pr.gov.br/modules/ conteudo/conteudo.php? conteudo $=3128>$. Acesso em: 14 set. 2016.

PENNA, M. L. F. Um desafio para a saúde pública brasileira: o controle do dengue Dengue control: a challenge for the public health system in Brazil. Cad. Saúde Pública, v. 19, n. 1, p. 305-309, 2003.

TAUIL, P. L. Dengue e febre amarela: epidemiologia e controle no Brasil. Revista da Sociedade Brasileira de Medicina Tropical, v. 20, n. s1, 1987.

Urbanização e ecologia do dengue Urbanization and dengue ecology. Cad Saúde Pública, v. 17, p. 99-102, 2001.

Controle de doenças transmitidas por vetores no sistema único de saúde. Informe Epidemiológico do SUS, v. 11, n. 2, p. 59-60, 2002.

Perspectivas de controle de doenças transmitidas por vetores no Brasil. Revista da Sociedade Brasileira de Medicina Tropical, v. 39, n. 3, p. 275-277, 2006. 
BRIGAGÃO, G. da S.; CORRÊA, N. A. B. 